\title{
Anti-GAD associated post-infectious cerebellitis after COVID-19 infection
}

\author{
Ahmed Serkan Emekli ${ }^{1}$ - Asuman Parlak ${ }^{1} \cdot$ Nejla Yılmaz Göcen ${ }^{2} \cdot$ Murat Kürtüncü $^{3}$
}

Received: 6 March 2021 / Accepted: 18 July 2021 / Published online: 30 July 2021

(c) Fondazione Società Italiana di Neurologia 2021

\begin{abstract}
The coronavirus disease 2019 (COVID-19), caused by the novel severe acute respiratory syndrome coronavirus 2 (SARS$\mathrm{CoV}-2$ ), continues to spread rapidly all over the world. Besides severe pneumonia, it causes multisystemic disease, including neurological findings. Here, we present a patient with anti-glutamic acid decarboxylase (anti-GAD) antibody-associated cerebellitis developed after COVID-19 infection. The patient responded well to the immune treatments. Our knowledge about SARS-CoV-2 infection-related neurological disorders is limited. New data are needed to recognize the clinical spectrum of autoimmune neurological disorders that emerges after SARS-CoV-2 infection.
\end{abstract}

Keywords Ataxia $\cdot$ Anti-GAD $\cdot$ COVID-19 $\cdot$ SARS-CoV-2 $\cdot$ Post-infectious $\cdot$ Cerebellitis

\section{Introduction}

The coronavirus disease 2019 (COVID-19), caused by the novel severe acute respiratory syndrome coronavirus 2 (SARS-CoV-2), continues to spread rapidly all over the world [1]. Besides severe pneumonia, it causes multisystemic disease, including neurological findings such as anosmia, cranial neuropathies, Guillain-Barre syndrome, and encephalitis. Our current knowledge about post-infectious immune pathologies caused by SARS-CoV-2 is limited. Herein, we present a patient with anti-glutamic acid decarboxylase (anti-GAD) antibody-associated cerebellitis developed after COVID-19 infection.

Ahmed Serkan Emekli

serkanemekli@gmail.com

1 Department of Neurology, Karabuk University Training and Research Hospital, Karabuk, Turkey

2 Department of Infectious Diseases, Karabuk University Training and Research Hospital, Karabuk, Turkey

3 Department of Neurology, Istanbul Faculty of Medicine, Istanbul University, Istanbul, Turkey

\section{Case}

A 54-year-old male teacher presented with anosmia and generalized myalgia that started 2 days ago. The patient's past medical history revealed primary hypertension treated with candesartan for 2 years. On admission, the patient did not have any respiratory symptoms, and his vital signs were within normal limits. There was pneumonic infiltration suggestive of asymptomatic pneumonia on his chest computed tomography. The patient's nasopharyngeal real-time reverse transcriptase-polymerase chain reaction (rt-PCR) test for SARS-CoV-2 was positive. He was treated with favipiravir with a loading dosage of $1600 \mathrm{mg}$ and maintenance dosage of $600 \mathrm{mg}$ per day, acetylsalicylic acid $100 \mathrm{mg}$ per day, and paracetamol $1000 \mathrm{mg}$ per day.

After treatment for 5 days, the patient's symptoms resolved. However, 2 weeks later, the patient complained of incoordination during writing due to a slight tremor in his hands. One week later, truncal ataxia was added to the clinical picture causing gait difficulty. On his first neurological examination in the emergency department, the patient was disoriented. He had dysarthria and a convergence spasm in his ophthalmologic examination. Deep tendon reflexes were normoactive, and he had bilateral moderate appendicular and severe truncal ataxia. He could not walk independently with a Scale for Assessment and Rating of Ataxia (SARA) score of 19.5/40. 
The patient's brain magnetic resonance imaging (MRI) revealed edematous changes and hyperintensities in the cerebellar cortex in T2-weighted and FLAIR images (Fig. 1). Additionally, mild pial contrast enhancement was also observed in the cerebellum. The patient's cerebrospinal fluid (CSF) examination revealed a normal opening pressure. There were 20 lymphocytes $/ \mathrm{mm}^{3}$ in the CSF. The CSF total protein level was $45 \mathrm{mg} / \mathrm{dl}$ (normal range: $15-45 \mathrm{mg} / \mathrm{dl}$ ); the glucose level was $62 \mathrm{mg} / \mathrm{dl}$ with a simultaneous blood glucose level of $97 \mathrm{mg} / \mathrm{dl}$. The CSF culture was sterile.

To investigate COVID-19 encephalitis, the SARS-CoV-2 rt-PCR test was repeated for both CSF and nasopharyngeal specimens with negative results. Additionally, thyroid function tests and serum vitamin $\mathrm{B}_{12}$ and folate levels were also within normal limits. However, anti-thyroid peroxidase, anti-thyroglobulin levels, and anti-tissue transglutaminase IgG were slightly higher. VDRL, wright test for Brucella infection, anti-tissue transglutaminase $\operatorname{IgA}$, anti-Hu, antiYo, anti-Ri, anti-amphiphysin, anti-Tr, anti-PCA-2, anti-Ma, anti-CV2-1, anti-ANNA-3, anti-NMDA-R, anti-AMPA-R1, anti-AMPA-R2, anti-Caspr2, anti-LGI1, and anti-GABA$\mathrm{R}$ antibodies were negative in the serum samples. Serum anti-GAD antibody level was $114.41 \mathrm{IU} / \mathrm{ml}$ (normal range: 0-5 IU/ml). We also performed a chest and abdominal CT scan to investigate further, which did not show any abnormality.

The patient was treated with methylprednisolone $1 \mathrm{gr} /$ day for 10 days and intravenous immunoglobulin $0.4 \mathrm{gr} / \mathrm{kg} /$ day for 5 days. One month after the treatment, the patient was able to walk independently without any signs of appendicular and truncal ataxia with a mild tremor in his upper extremities that was successfully treated with propranolol. Monthly intravenous immunoglobulin and oral methylprednisolone treatment were given for 3 months. The patient's SARA score 3 months after his first symptoms was $1 / 40$.

\section{Discussion}

Immune-mediated neuronal apoptosis and dysfunction are observed in the autoimmune cerebellar syndromes, including gluten ataxia, opsoclonus-myoclonus syndrome, paraneoplastic cerebellar degeneration, and post-infectious cerebellar syndromes using various mechanisms [2]. One of the well-defined ataxic syndromes, the anti-GAD antibody, may cause an autoimmune cerebellar syndrome by impairing GABAergic transmission via cell-mediated immunity [3].

As far as our knowledge, this is the first case with post-infectious anti-GAD antibody-related cerebellar syndrome after SARS-CoV-2 infection. Reported cases of ataxia associated with SARS-CoV-2 are reviewed in Table 1. Para-/post-infectious ataxia is reported between 7 and 83 years of age. However, the majority of cases were reported in middle-aged male patients as in our case. Besides ataxia, a wide spectrum of clinical findings was observed including opsoclonus, myoclonus, ocular movement disorders, seizures, vertigo, behavioral disorders, involuntary movements, tremor, and dysarthria. Our case adds convergence spasm to these diverse findings. Four out of 31 cases reviewed in Table 1 have abnormal brain imaging including hyperintensities in the brainstem and cerebellum. However, brain FDG-PET abnormalities in the frontal cortex and cerebellum were reported in another three patients. Similar to our case, bilateral cerebellar hemispheres and vermis hyperintensities in FLAIR imaging and cerebellar cortical meningeal contrast enhancement were observed by Fadakar et al. [25]. In contrarily to our case, the presentation of cerebellar ataxia was concomitant with COVID-19 infection, and SARS-CoV-2 rt-PCR test was found positive in CSF [25]. Although autoantibody screening was performed in the majority of cases, anti-amphiphysin, anti-NMDAR antibodies, and autoantibodies directed against the nuclei of
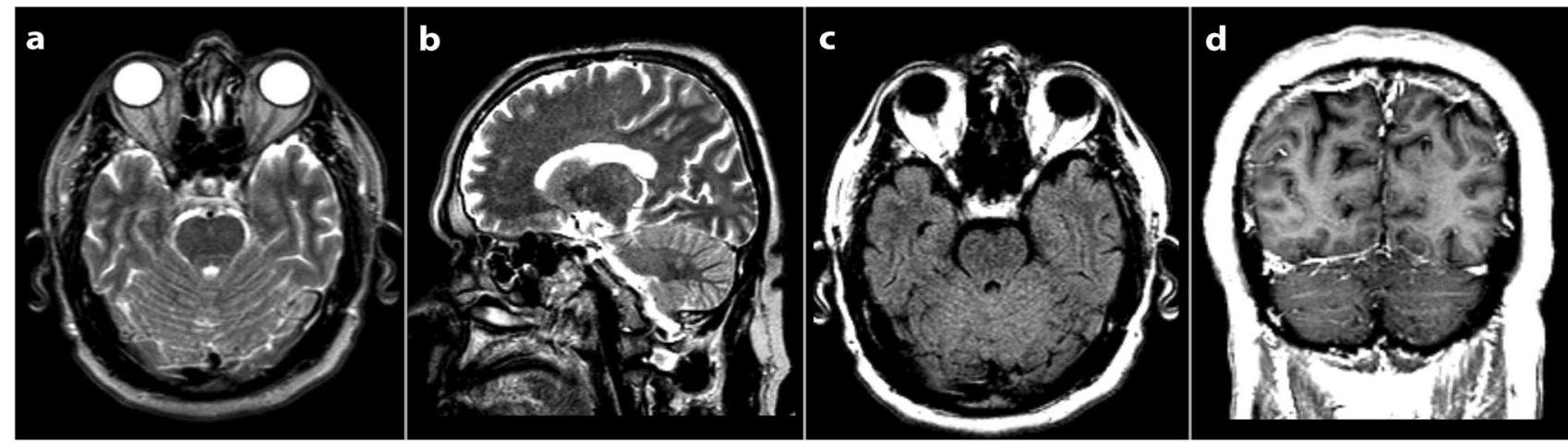

Fig. 1 Brain MRIs show edematous hyperintense changes in T2-weighted and FLAIR images in the cerebellum (a, b, c) and cerebellar pial contrast enhancement $(\mathbf{d})$ 


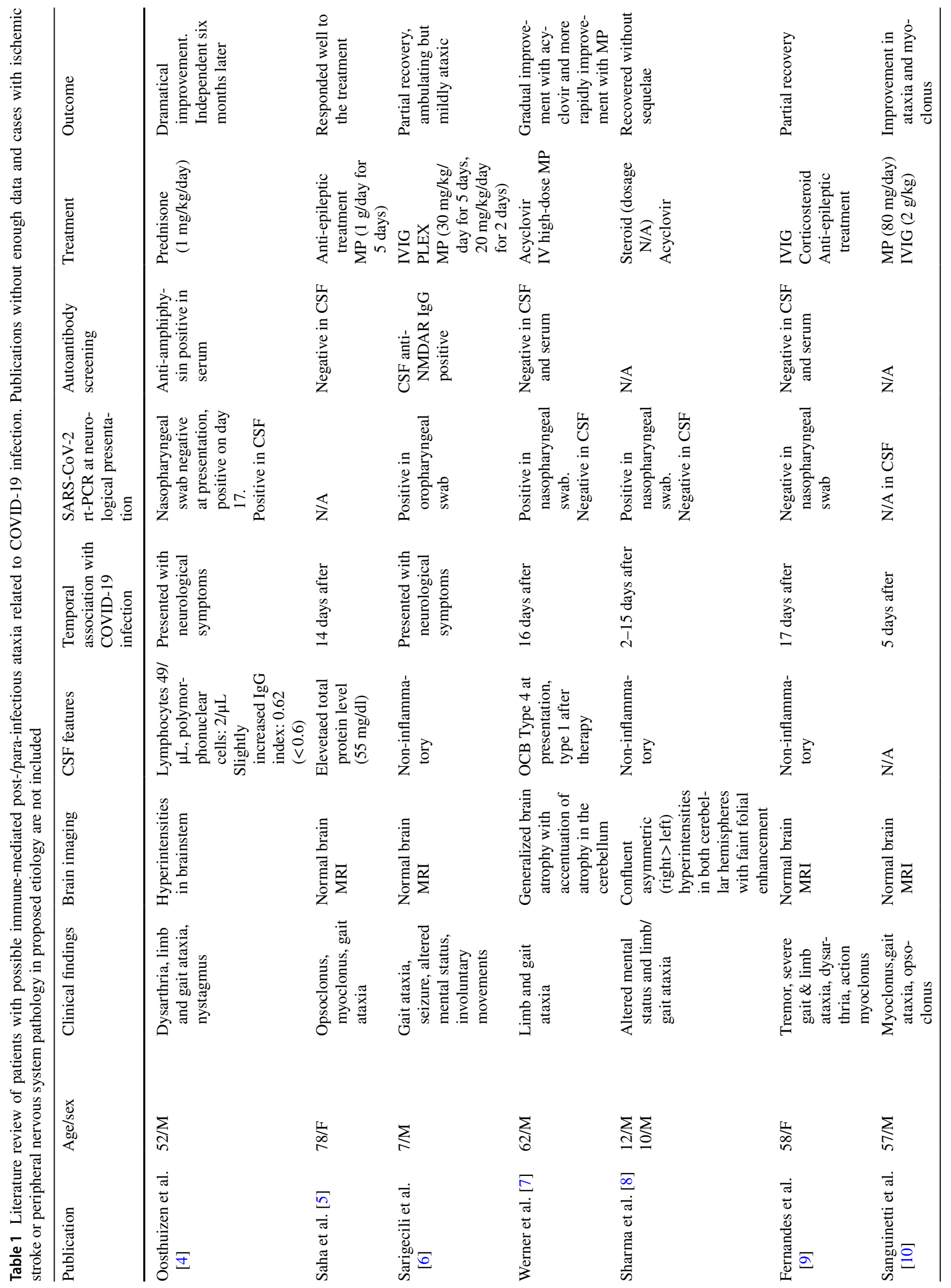




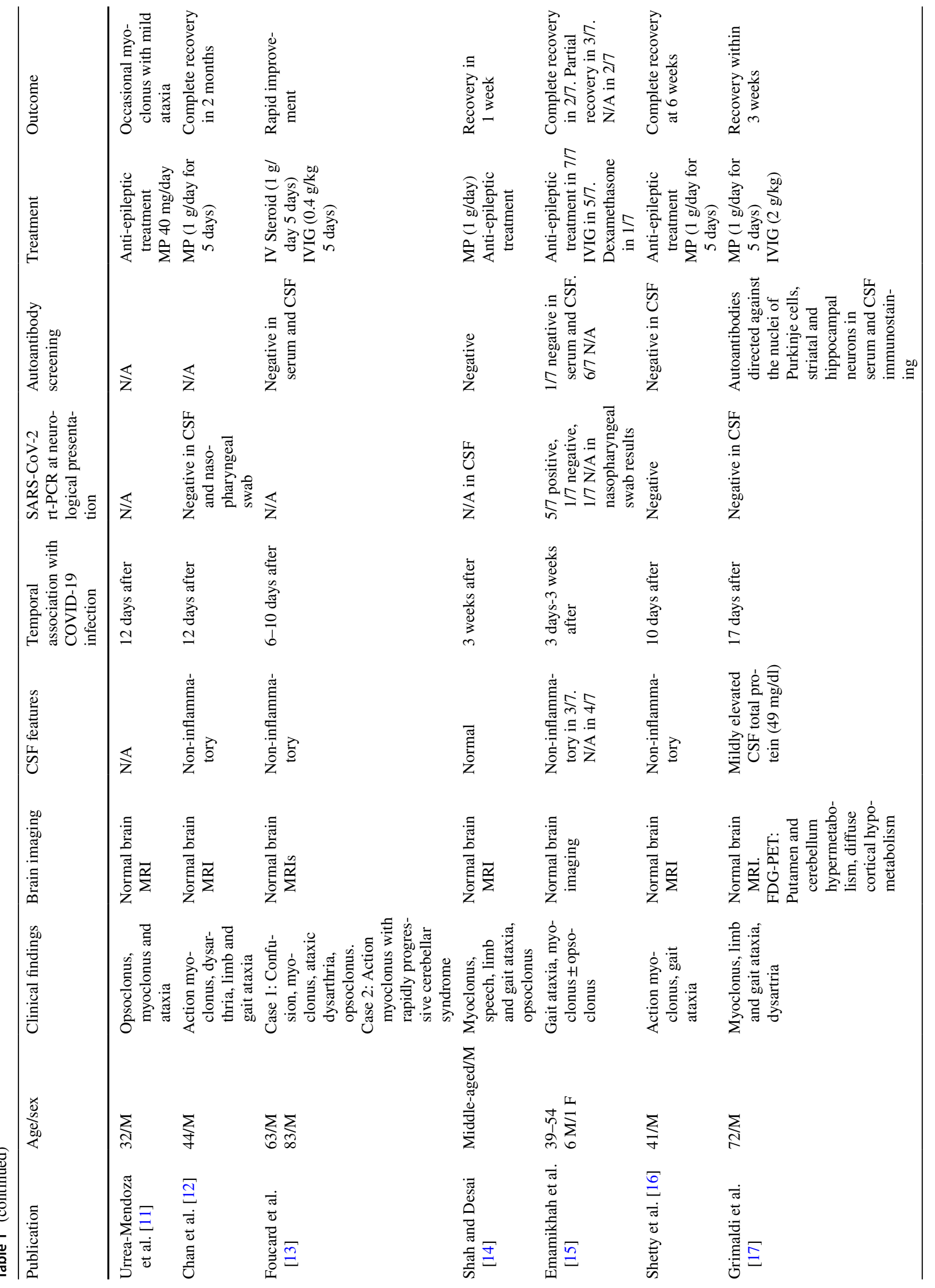




\begin{tabular}{|c|c|c|c|c|c|}
\hline 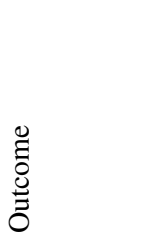 & 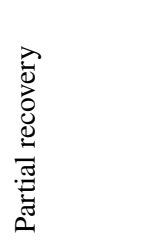 & 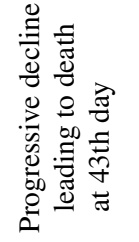 & 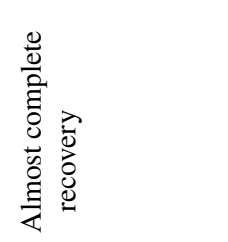 & 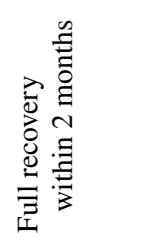 & 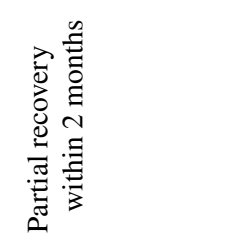 \\
\hline 苂 & 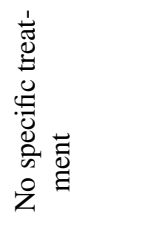 & 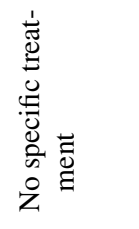 & 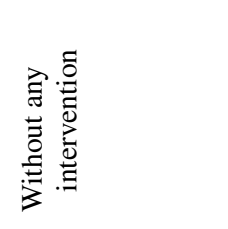 & 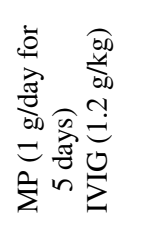 & 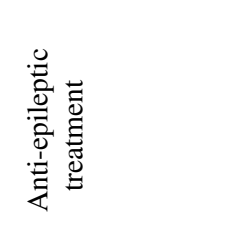 \\
\hline 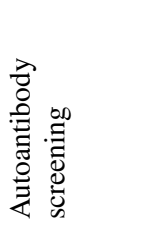 & 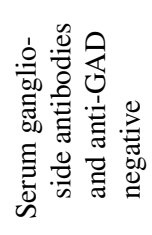 & $\overleftrightarrow{\mathrm{z}}$ & $\overleftrightarrow{\mathrm{z}}$ & 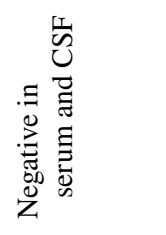 & 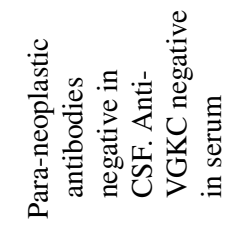 \\
\hline 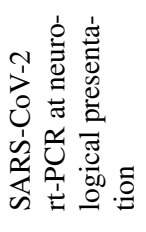 & 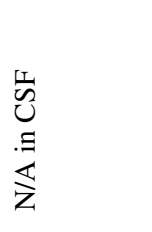 & 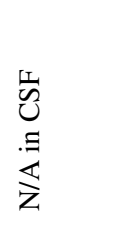 & 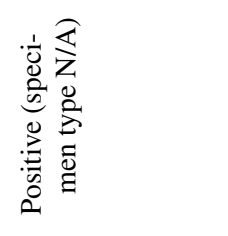 & 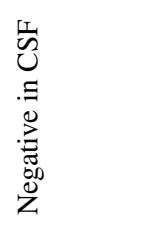 & 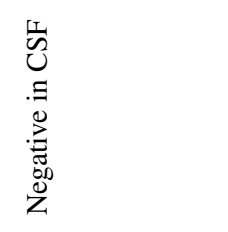 \\
\hline 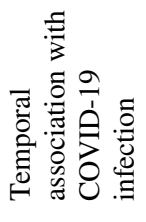 & 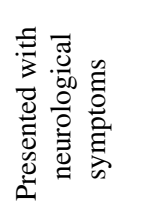 & 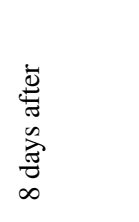 & 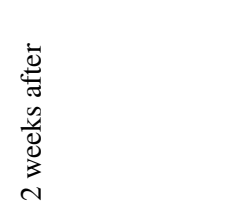 & 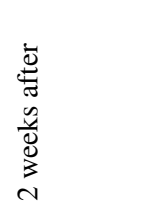 & 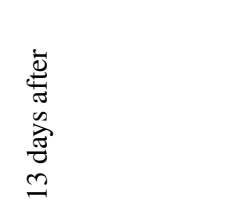 \\
\hline 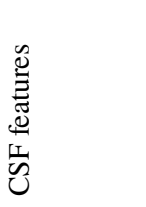 & 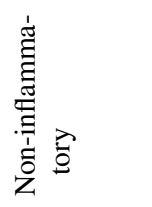 & $\overleftrightarrow{\mathbb{Z}}$ & $\overleftrightarrow{\mathbb{Z}}$ & 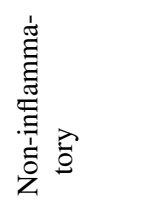 & 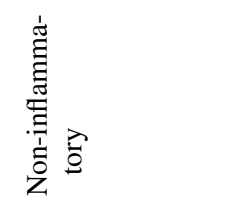 \\
\hline 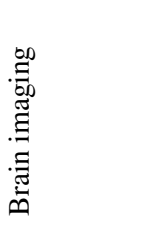 & 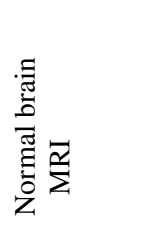 & 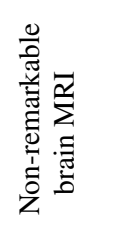 & 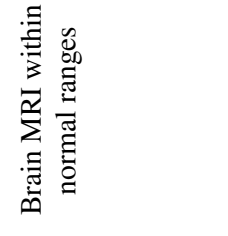 & 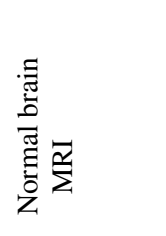 & 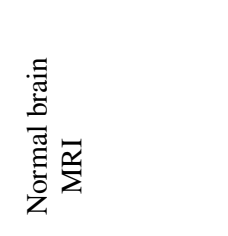 \\
\hline 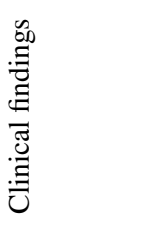 & 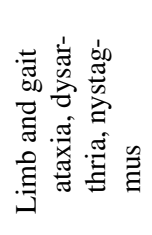 & 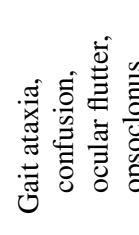 & 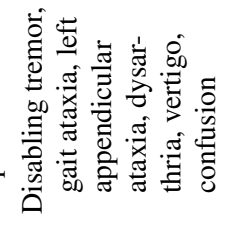 & 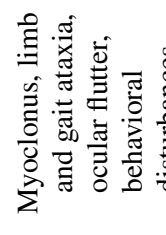 & 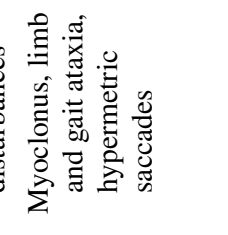 \\
\hline 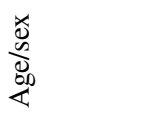 & 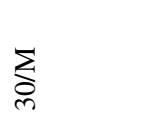 & $\underset{\Omega}{\Sigma}$ & $\underset{\text { in }}{\sum_{0}}$ & $\sum_{\underset{f}{f}}$ & $\sum_{\substack{++}}$ \\
\hline 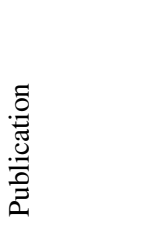 & 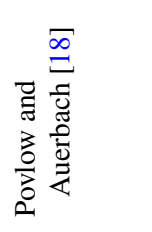 & 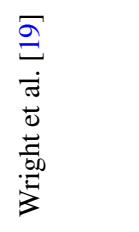 & 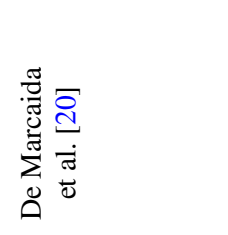 & 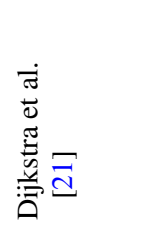 & 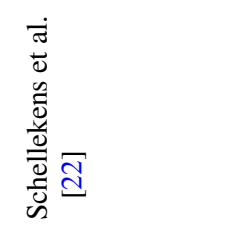 \\
\hline
\end{tabular}




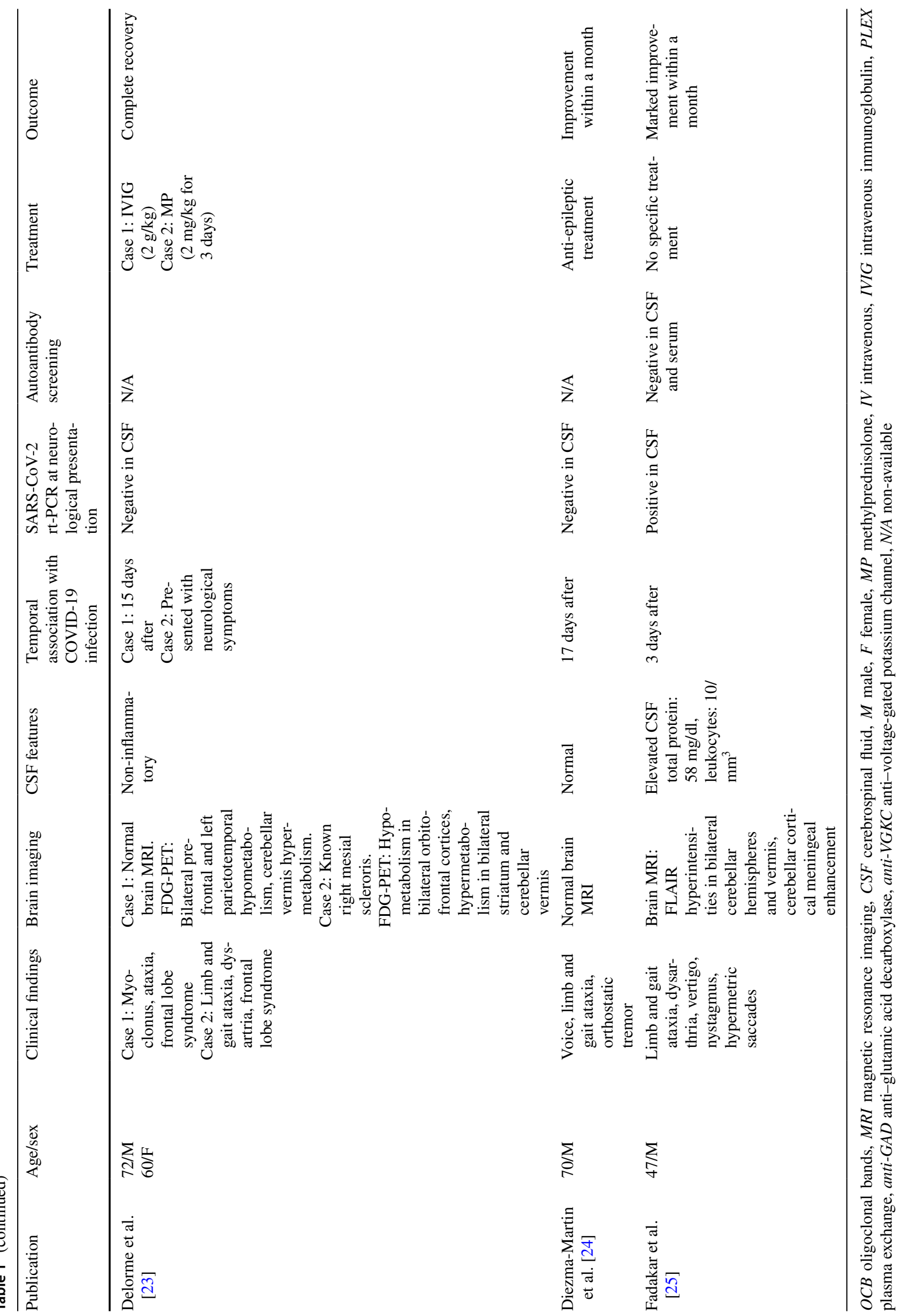


Purkinje cells, striatal and hippocampal neurons in serum, and CSF immunostaining were reported only in three cases $[4,6,17]$. In the reported cases in which patients who had SARS-CoV-2 rt-PCR test were positive either in CSF or nasopharyngeal swabs, it indicates cerebellar syndrome is related to the infectious process. The majority of cases responded well to the immunotherapy, although mortality was reported in one patient without specific treatment.

In our case, the SARS-CoV-2 rt-PCR test was negative in the nasopharyngeal and CSF specimens, whereas anti-GAD antibody was detected with a high titer in the etiological workup of the cerebellar syndrome. It has been reported that the detection of anti-GAD antibodies in high titers suggests autoantibody-specific disease [2]. The dramatic response to immune therapies such as high-dose steroids and intravenous immunoglobulin also suggests the existence of an underlying autoimmune process. Besides, anti-GAD-associated neurological disorders are frequently accompanied by autoimmune disorders such as autoimmune thyroiditis and gluten sensitivity, as in our case [26]. Various side effects are reported with high-dose favipiravir in the treatment of COVID-19 [27]. However, cerebellar ataxia and convergence spasm are not among well-known adverse effects of favipiravir use, and drug toxicity is not a likely cause in our case.

These findings confirm that high titer anti-GAD seropositivity is associated with post-infectious cerebellar syndrome in our case. Besides our findings, anti-amphiphysin, antiCaspr2, anti-GD1b, and anti-NMDAR antibodies related to neurological disorders after SARS-CoV-2 infection have been reported in the literature, suggesting that SARS-CoV-2 infection might trigger autoimmunity [4, 6, 28-30]. However, it seems complicated to establish a direct pathogenetic relationship between SARS-CoV-2 infection and anti-GADassociated autoimmune cerebellitis.

\section{Conclusion}

Since the first months of its emergence, SARS-CoV-2 infection has been associated with a wide array of neurological and neuropsychiatric findings, including encephalitis, inflammatory central nervous system syndromes, ischemic strokes, and peripheral neurological diseases [31]. Our knowledge about SARS-CoV-2 infection-related neurological disorders is limited. New data are needed to recognize the clinical spectrum of autoimmune neurological disorders that emerges after SARS-CoV-2 infection.

Author contributions ASE and MK designed the study; ASE, AP, and NYG assembled the data. All authors wrote and approved the final article.

\section{Declarations}

Ethical approval All procedures performed in studies involving human participants were in accordance with the ethical standards of the institutional and/or national research committee and with the 1964 Helsinki declaration and its later amendments or comparable ethical standards. Informed consent to publish was obtained from the participant. Ethics committee approval was not applicable as the data was analyzed retrospectively and had no effect on treatment.

Conflict of interest The authors declare no competing interests.

\section{References}

1. Wiersinga WJ, Rhodes A, Cheng AC, Peacock SJ, Prescott HC (2020) Pathophysiology, transmission, diagnosis, and treatment of coronavirus disease 2019 (COVID-19): a review. JAMA 324:782-793

2. Mitoma H, Manto M, Hampe CS (2018) Immune-mediated cerebellar ataxias: practical guidelines and therapeutic challenges. Curr Neuropharmacol 17:33-58

3. Manto M, Mitoma H, Hampe CS (2019) Anti-GAD antibodies and the cerebellum: where do we stand? Cerebellum 18:153-156

4. Oosthuizen K, Steyn EC, Tucker L, Ncube IV, Hardie D, Marais S (2021) SARS-CoV-2 encephalitis presenting as a clinical cerebellar syndrome: a case report. Neurology. (Online ahead of print). https://doi.org/10.1212/WNL.0000000000012051.

5. Saha B, Saha S, Chong WH (2021) 78-year-old woman with opsoclonus myoclonus ataxia syndrome secondary to COVID-19. BMJ Case Rep. 14:e243165.

6. Sarigecili E, Arslan I, Ucar HK, Celik U (2021) Pediatric antiNMDA receptor encephalitis associated with COVID-19. Child's Nerv Syst. 1-4 (Online ahead of print). doi: https://doi.org/10. 1007/s00381-021-05155-2.

7. Werner J, Reichen I, Huber M, Abela IA, Weller M, Jelcic I (2021) Subacute cerebellar ataxia following respiratory symptoms of COVID-19: a case report. BMC Infect Dis 21:1-7

8. Sharma S, Ruparelia J, Bhaskar S, Tiwari S, Nag VL, Panda S (2021) Acute fulminant cerebellitis in children with COVID19 infection: a rare but treatable complication. Pediatr Neurol 119:45-47

9. Fernandes J, Puhlmann P (2021) Opsoclonus myoclonus ataxia syndrome in severe acute respiratory syndrome coronavirus-2. J Neurovirol. 2-4 (Online ahead of print). doi: https://doi.org/10. 1007/s13365-021-00974-0.

10. Sanguinetti SY, Ramdhani RA (2021) Opsoclonus-myoclonusataxia syndrome related to the novel coronavirus (COVID-19). J Neuro-Ophthalmology (Online ahead of print). doi: https://doi. org/10.1097/WNO.0000000000001129.

11. Urrea-Mendoza E, Okafor K, Ravindran S, Absher J, Chaubal V, Revilla FJ (2021) Opsoclonus-myoclonus-ataxia syndrome (Omas) associated with sars-cov-2 infection: post-infectious neurological complication with benign prognosis. Tremor and Other Hyperkinetic Movements 11:1-4

12. Chan JL, Murphy KA, Sarna JR (2021) Myoclonus and cerebellar ataxia associated with COVID-19: a case report and systematic review. J. Neurol. 1-32 (Online ahead of print). doi: https://doi. org/10.1007/s00415-021-10458-0.

13. Foucard C, San-Galli A, Tarrano C, Chaumont H, Lannuzel A, Roze E (2021) Acute cerebellar ataxia and myoclonus with or without opsoclonus: a parainfectious syndrome associated with COVID-19. Eur J Neurol. 0-3 (Online ahead of print). doi: https:// doi.org/10.1111/ene.14726. 
14. Shah PB, Desai SD (2021) Opsoclonus myoclonus ataxia syndrome in the setting of COVID-19 infection. Neurology 96:33

15. Emamikhah M, Babadi M, Mehrabani M, Jalili M, Pouranian M, Daraie $\mathrm{P}$ et al (2021) Opsoclonus-myoclonus syndrome, a postinfectious neurologic complication of COVID-19: case series and review of literature. J Neurovirol 27:26-34

16. Shetty K, Jadhav AM, Jayanthakumar R, Jamwal S, Shanubhogue T, Reddy MP et al (2021) Myoclonus-ataxia syndrome associated with COVID-19. J Mov Disord 14:153-156

17. Grimaldi S, Lagarde S, Harlé JR, Boucraut J, Guedj E (2020) Autoimmune encephalitis concomitant with SARS-CoV-2 infection: insight from 18F-FDG PET imaging and neuronal autoantibodies. J Nucl Med 61:1726-1729

18. Povlow A, Auerbach AJ (2021) Acute cerebellar ataxia in COVID19 infection: a case report. J Emerg Med 60:73-76

19. Wright D, Rowley R, Halks-Wellstead P, Anderson T, Wu TY (2020) Abnormal saccadic oscillations associated with severe acute respiratory syndrome coronavirus 2 encephalopathy and ataxia. Mov Disord Clin Pract 7:980-982

20. de Marcaida JA, Lahrmann J, Machado D, Bluth L, Dagostine M, Moro-de Casillas M et al (2020) Clinical characteristics of coronavirus disease 2019 (COVID-19) among patients at a movement disorders center. Geriatr 5:54

21. Dijkstra F, Van den Bossche T, Willekens B, Cras P, Crosiers D (2020) Myoclonus and cerebellar ataxia following COVID-19. Mov Disord Clin Pract 7:974-976

22. Schellekens MMI, Bleeker-Rovers CP, Keurlings PAJ, Mummery CJ, Bloem BR (2020) Reversible myoclonus-ataxia as a postinfectious manifestation of COVID-19. Mov Disord Clin Pract 7:977-979

23. Delorme C, Paccoud O, Kas A, Hesters A, Bombois S, Shambrook P et al (2020) COVID-19-related encephalopathy: a case series with brain FDG-positron-emission tomography/computed tomography findings. Eur J Neurol 27:2651-2657

24. Diezma-Martín AM, Morales-Casado MI, García Alvarado N, Vadillo Bermejo A, López-Ariztegui N, Sepúlveda Berrocal MA
(2020) Tremor and ataxia in COVID-19. Neurologia (Engl Ed) 35:409-410

25. Fadakar N, Ghaemmaghami S, Masoompour SM, Shirazi Yeganeh B, Akbari A, Hooshmandi S et al (2020) A first case of acute cerebellitis associated with coronavirus disease (COVID-19): a case report and literature review. Cerebellum 19:911-914

26. Dade M, Berzero G, Izquierdo C, Giry M, Benazra M, Delattre JY et al (2020) Neurological syndromes associated with anti-gad antibodies. Int J Mol Sci 21:3701

27. Kaur RJ, Charan J, Dutta S, Sharma P, Bhardwaj P, Sharma P et al (2020) Favipiravir use in COVID-19: Analysis of suspected adverse drug events reported in the WHO database. Infect Drug Resist 13:4427-4438

28. Panariello A, Bassetti R, Radice A, Rossotti R, Puoti M, Corradin $M$ et al (2020) Anti-NMDA receptor encephalitis in a psychiatric Covid-19 patient: A case report. Brain Behav Immun 87:179-181

29. Monti G, Giovannini G, Marudi A, Bedin R, Melegari A, Simone AM et al (2020) Anti-NMDA receptor encephalitis presenting as new onset refractory status epilepticus in COVID-19. Seizure $81: 18-20$

30. Guilmot A, Maldonado Slootjes S, Sellimi A, Bronchain M, Hanseeuw B, Belkhir L et al (2021) Immune-mediated neurological syndromes in SARS-CoV-2-infected patients. J Neurol 268:751-757

31. Paterson RW, Brown RL, Benjamin L, Nortley R, Wiethoff S, Bharucha $T$ et al (2020) The emerging spectrum of COVID-19 neurology: clinical, radiological and laboratory findings. Brain $143: 3104-3120$

Publisher's note Springer Nature remains neutral with regard to jurisdictional claims in published maps and institutional affiliations. 